\title{
Care pathways for common infectious diseases
}

\author{
H Grant Stiver MD FRCPC ${ }^{1}$, Division of Infectious Diseases, Department of Medicine, University of British Columbia \\ and Vancouver Hospital and Health Sciences Centre, Vancouver, British Columbia \\ John M Conly MD FRCPC ${ }^{\top}$, University Health Network, University of Toronto, Toronto, Ontario
}

$\mathrm{C}^{\mathrm{a}}$ are pathways and maps are attractive strategies for dealing with various medical conditions because they can be formulated by experts, are algorithmic in nature, are easy to use by busy physicians, and facilitate the practice of an optimal standard of care (1-3). Although infectious diseases rank as one of the most common types of illnesses encountered, few detailed care pathways have been produced or published. In this supplement, care pathways for several relatively common infections are presented. In many conditions, parenteral antibiotic regimens may be required, and these regimens can be administered on an outpatient basis in many cases. Outpatient intravenous antibiotic therapy, administered through a local hospital emergency room, at a daycare facility or in the patient's residence, has long been shown to be as safe and efficacious as parenteral therapy administered in hospital, is considerably less expensive per se and allows greater efficiency in the use of hospital beds, making beds available for other patients (4). It has become a common treatment modality for patients with certain acute and chronic infectious diseases (5).

In other cases, oral antibiotics may be equally efficacious, available at less cost and more convenient for the patient. Oral agents are often used as a stepdown therapy after acute symptoms have been controlled with parenteral therapy (6). A decision to use oral therapy depends on the pharmacological characteristics of the antibiotic, ie, it must have sufficient bioavailability and dose tolerability to support adequately high serum and tissue concentrations, and the patient must be compliant in taking the oral medications. Theoretically, if these conditions are met and careful follow-up is assured, even though the infections may manifest acute symptoms, oral therapy constitutes an efficacious regimen, and from a purely bacteriological point of view, there is no absolute requirement for intravenous antibiotic administration. In most 'real life' clinical settings, however, the severity of the patient's illness determines whether intravenous or oral therapy is begun. Acute streptococcal cellulitis is a good example of this. In this situation, one may have an uncomplicated focal limb infection with a very susceptible organism that is pharmacologically amenable to serum levels that can be obtained with oral antibiotics such as cephalexin or clindamycin. However, intravenous therapy is often started initially because the patient's host response makes him or her appear very sick. In patients with endocarditis, there are dose limitations for most oral regimens and very little clinical data on their efficacy; in these cases, the intravenous route is preferred for a different reason. Most care pathways presented in this issue provide an intravenous-to-oral switch strategy when the infection is well under control and alternate recommendations for beta-lactam allergic patients.

Management strategies for infectious diseases do undergo changes with the advent of newer antimicrobial agents and with the need to keep pace with the fundamental changes in medical care delivery that are occurring across the country. The following articles present care pathways, including recommendations for antimicrobial therapy for bacterial endocarditis (pages 4D-10D), acute cellulitis (pages 11D-14D), diabetic foot infection (pages 15D-21D), community-acquired pneumonia (pages 22D-26D), febrile neutropenia (pages 27D-33D), and septic arthritis and osteomyelitis (pages 34D-40D). These pathways should be viewed as guidelines to assist individuals in developing policies and standards of care for the populations that they serve within their own regions and jurisdictions.

\section{REFERENCES}

1. Ellis BW, Johnson S. The care pathway: a tool to enhance clinical governance. Clin Perform Qual Health Care 1999;7:134-44.

2. Kelly CS, Andersen CL, Pestian JP, et al. Improved outcomes for hospitalized asthmatic children using a clinical pathway. Ann Allergy Asthma Immunol 2000;84:509-16.

3. Marie TJ, Lau CY, Wheeler SL, et al. A controlled trial of a critical pathway for treatment of community-acquired pneumonia. JAMA 2000;283:749-55.

4. Stiver HG, Telford GO, Mossey JM, et al. Intravenous antibiotic therapy at home. Ann Intern Med 1978;89:690-3.

5. Tice AD. Handbook of Outpatient Parenteral Therapy. New York: Scientific American Inc, 1997:122.

6. Lew DP, Waldvogel FA. Osteomelitis. N Engl J Med 1997;336:999-1007. 


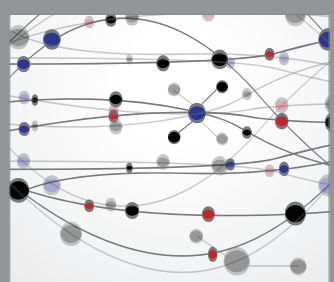

The Scientific World Journal
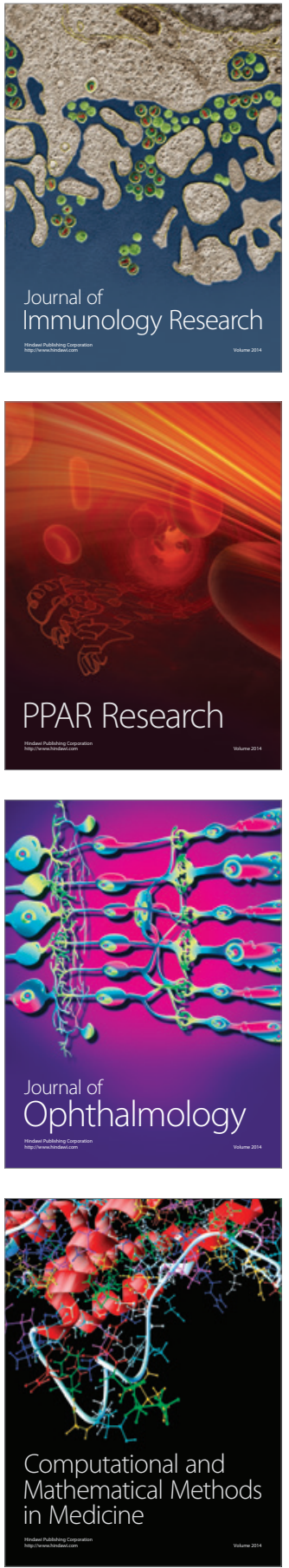

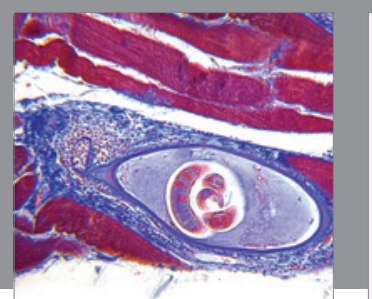

Gastroenterology Research and Practice

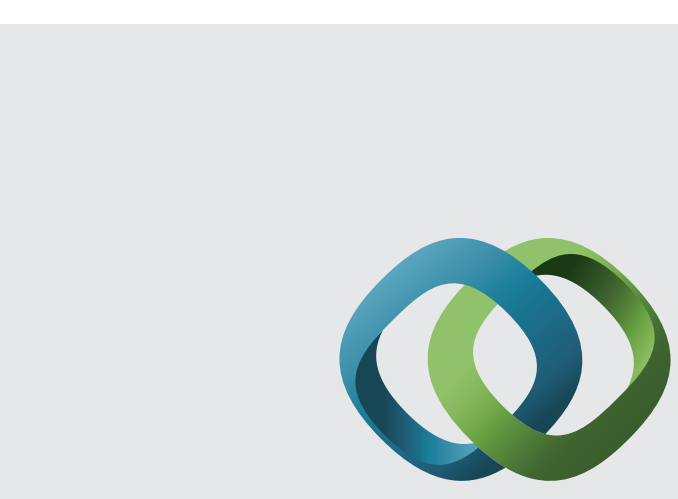

\section{Hindawi}

Submit your manuscripts at

http://www.hindawi.com
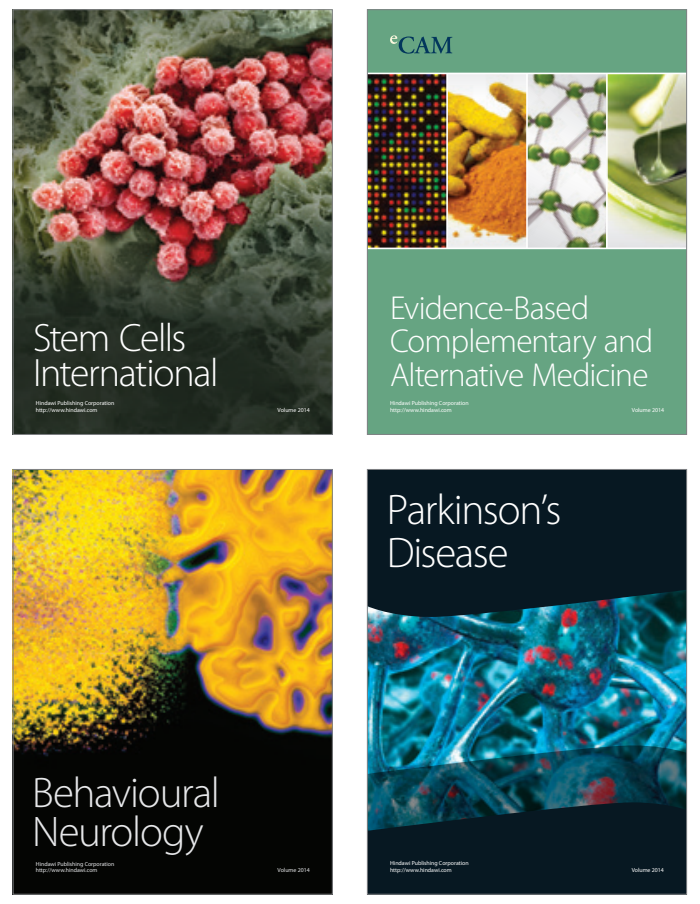
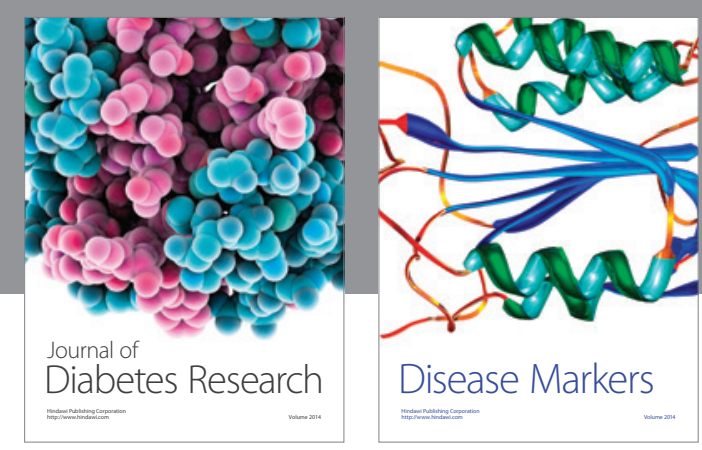

Disease Markers
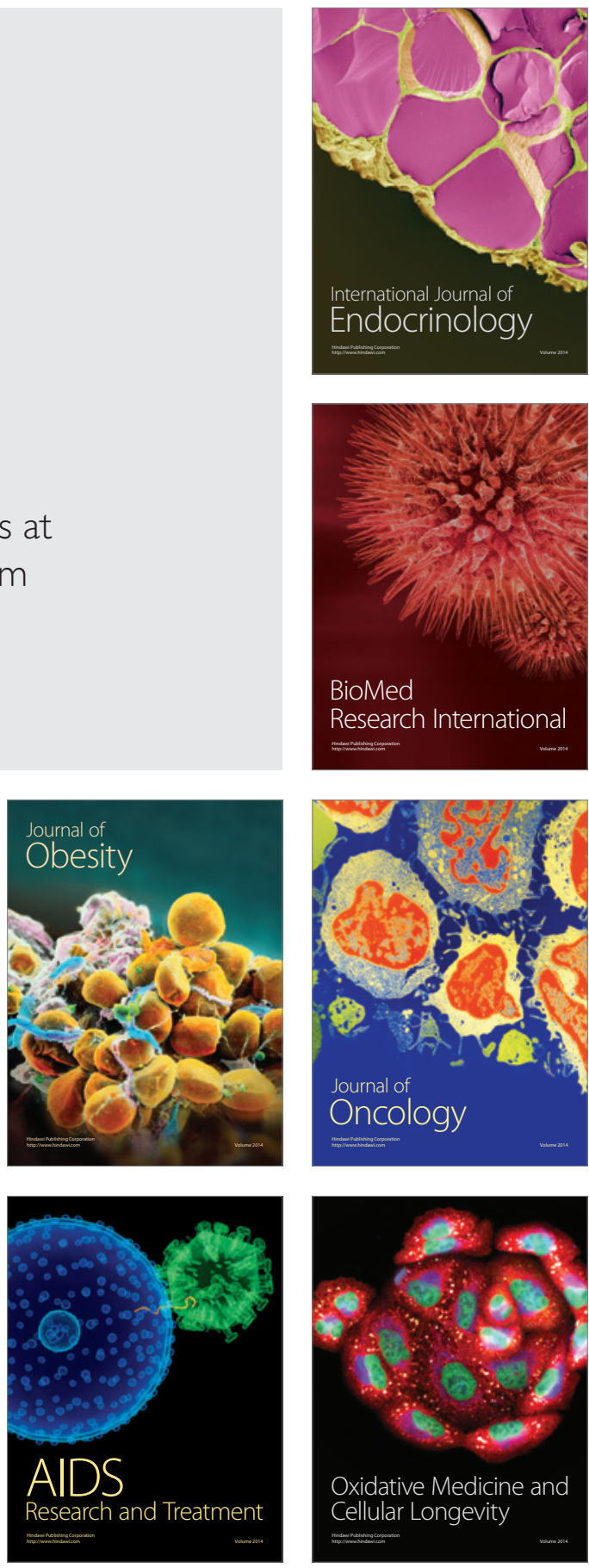